\title{
Temporal Lobe Epilepsy Surgical Outcome Prediction
}

\author{
Simon Duchesne, Neda Bernasconi, Andrea Bernasconi, and D. Louis Collins \\ Montréal Neurological Institute (MNI), McGill Univ., Montréal, Canada H3A 2B4 \\ \{duchesne,neda, andrea, louis\}@bic.mni.mcgill.ca
}

\begin{abstract}
We wished to study pre-operative T1-weighted MRI of intractable temporal lobe epilepsy (TLE) patients who had undergone selective amygdala-hippocampectomy as part of their surgical treatment. We performed a voxel-based morphometry study of gray and white matter (GM,WM) concentration changes by comparing TLE patients with positive and negative surgical outcome. GM concentration changes were primarily located in the left lateral temporal neocortical region, while more extensive changes were found in left lateral temporal and occipital WM. Using those areas to define a region of interest, we showed that mean GM and WM concentration for all voxels within that region can be used to predict surgical outcome with $97 \%$ accuracy.
\end{abstract}

Keywords: Temporal Lobe Epilepsy, Surgical Outcome, Voxel-Based Morphometry, Grey Matter Atrophy, White Matter Atrophy, Linear Discriminant Analysis

\section{Introduction}

Predicting surgical outcome in the treatment of temporal lobe epilepsy (TLE) is an outstanding challenge. Some authors have attempted to find neuroimaging anatomical markers that can be used for that purpose, but no completely reliable indicator has been found to date.

The standard neurosurgical procedure in TLE consists in resection of the hippocampus and often parts of the neighboring structures, including portions of the amygdala and parahippocampal gyrus. Post-surgical outcome can be characterized as either positive (complete remission and disappearance of all seizures) or negative (all levels of complications). While the majority of patients undergoing surgery have positive outcome, it is impossible at present to determine a priori if the procedure will be successful.

It is now accepted that the hippocampus is not the only structure affected in TLE, as grey matter (GM) and white matter (WM) atrophy in temporal and extra temporal areas have been repeatedly demonstrated. Voxel-based morphometry (VBM) [1 analyses of T1-weighted (T1w) MRI in large groups of patients with TLE can reliably detect and localize regions of GM and WM atrophy associated with the disease [2] 3] [4]. 
Our research hypothesis is that there exists areas that are linked to postsurgical outcome. The first step in our study was to identify those regions by comparing pre-operative MR volumes of patient cohorts having undergone surgery with positive and negative outcome. Our purpose is to explore whether surgical outcome is related to a pattern of GM or WM changes in TLE.

The second part of our work was an attempt at predicting surgical outcome based on simple image features. Our hypothesis is that in the regions of interest delineated by between-group analysis, there exists differences in GM or WM concentration, which can be used for classification purposes.

\section{Subjects}

The study population consisted of 39 consecutive patients with intractable, non-foreign-tissue TLE. Lateralization of seizure focus in TLE patients was determined by a comprehensive evaluation including prolonged videoelectroencephalogram (EEG) telemetry. The EEG focus was defined as right or left if more than $70 \%$ of seizures were recorded from one side. Manual MRI volumetry showed hippocampal atrophy ipsilateral to the seizure focus in all patients. Patients with left or right hippocampal atrophy were present in both groups.

Pre-operative T1w MR 3D images were acquired on a 1.5 T scanner using a T1-fast field echo sequence. All global MRI data were processed to correct for intensity non-uniformity due to scanner variations [5], linearly registered into stereotaxic space and resampled onto a $1 \mathrm{~mm}$ isotropic grid [6].

All patients underwent selective amygdala-hippocampectomy. The postoperative follow-up was at least 12 months for all patients, on which basis they were consolidated in two outcome groups: seizure free (positive outcome, $n=25$ ) or not seizure free (negative outcome, $n=14$ ).

\section{Methods}

\subsection{Detection of Atrophy Areas Related to Surgical Outcome}

Our primary objective was to determine areas of GM and WM atrophy related to TLE surgical outcome. To this end we performed between-group VBM analyses of GM and WM density maps, comparing negative outcome TLE patients to positive outcome patients.

The density maps were obtained after classification of T1w MR volumes [7], linearly registered 6 to a common reference target of 152 young healthy volunteers (ICBM 152 symmetrical average [8]). GM or WM concentration maps were blurred using an isotropic Gaussian kernel of $5 \mathrm{~mm}$ full-width at half-maximum. In those smoothed concentration maps, each voxel takes on a concentration value between $(0,1)$ indicative of the presence or not of GM or WM in that voxel. Tstatistics maps were obtained by estimating a generalized linear model at each voxel. Significant clusters, composed of a number or extent $E$ of resels above a 
cluster threshold t-value, were obtained following the procedure developed by Worsley et al. 9] to correct for data nonuniformity.

\subsection{Surgical Outcome Prediction}

We used the results from the preceding VBM analysis to define as a region of interest the ensemble of significant clusters. Our hypothesis for predicting surgical outcome is that in this area there exists differences in GM or WM concentration, which can be exploited for classification purposes.

Two measures were developed to capture these differences for all subjects. The first measure was a straightforward calculation of the mean GM or WM concentration of all region-of-interest voxels $j$, where $N_{j}$ is the number of such voxels. Thus, for subject $i$, with concentration map $[G M]_{i}$ for grey mater, the measure $M_{i}$ was calculated as follows:

$$
\mathbf{M}_{\mathbf{i}}=\frac{\sum[G M]_{i j}}{N_{j}}
$$

and a similar measure can be derived for the WM. For the second measure the t-value of the voxel in the between-group comparison volume was multiplied with the GM or WM concentration, thereby adding a weighting factor to the calculation. Thus, for subject $i$, the measure $W_{i}$ was calculated as follows:

$$
\mathbf{W}_{\mathbf{i}}=\sum_{j} C_{i j} \times V_{i j}
$$

where $V_{i}$ is the between-group t-stat volume, $C_{i}$ is the GM or WM concentration map for subject $i$, and each $j$ is a voxel belonging to the ensemble of significant clusters determined in section 3.1 .

For each of our four measures (GM/WM; mult/mean), linear discriminant analysis was used as a classifier for our two states, positive or negative outcome, using SYSTAT 10.2 (SSI, Point Richmond, CA).

\section{Results}

\subsection{Atrophy Areas Related to Surgical Outcome}

Figures 1 and 2 present the results of the between-group VBM analyses for GM and WM atrophy, respectively, for 14 negative outcome subjects compared to 25 positive outcome subjects. All voxels displayed are above the cluster threshold level of significance $(P<0.05$, corrected for data nonisotropy and multiple comparisons) and shown using a glass-brain approach similar to SPM 1. GM differences are detected primarily in the left lateral temporal neocortical areas. WM atrophy is more extensive, with contributions from left lateral temporal and occipital white matter. 


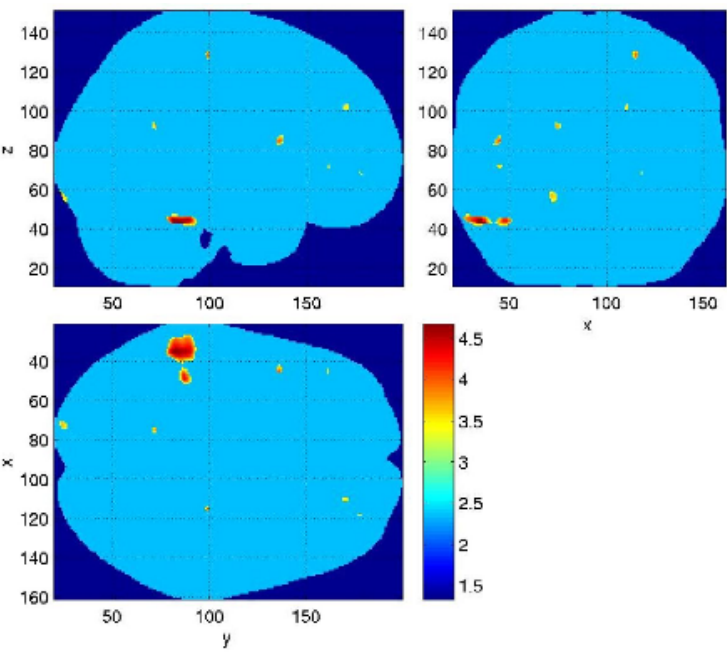

Fig. 1. GM atrophy for negative outcome group when compared to positive outcome group as determined by VBM, shown overlaid on a glass-brain. All voxels belong to significant clusters $(P<0.05$, corrected for data nonisotropy and multiple corrections). The left lateral temporal neocortical area seems particularly particularly affected.

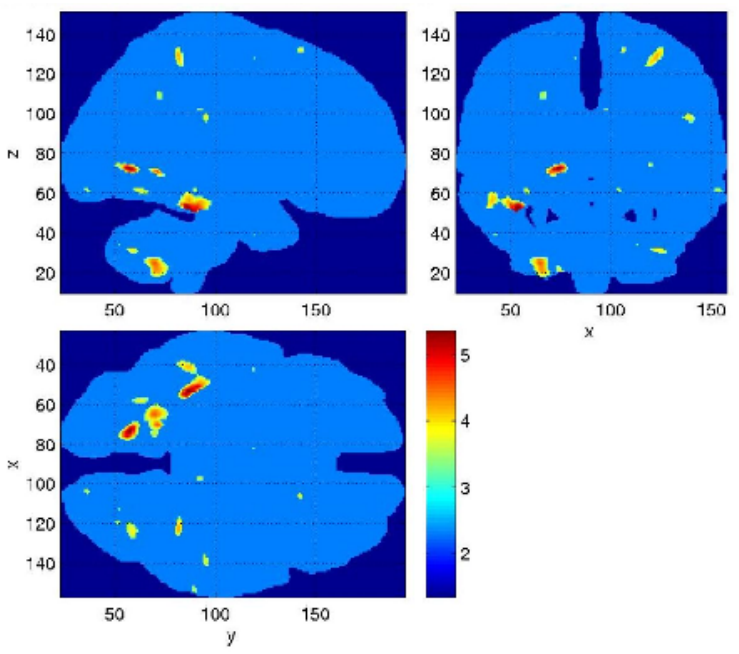

Fig. 2. WM atrophy map for negative outcome group when compared to positive outcome group as determined by VBM, shown overlaid on a glass-brain using the same parameters as Fig. 1. More areas seem related to surgical outcome, with predominance in the left lateral temporal and occipital white matter.

\subsection{Predicting Surgical Outcome}

Box plots of results for our two similarity measures are shown in Figure 3 (a) to (d). These show GM and WM measures taken for the average GM or WM concentration (mean) and t-stat weighted sum (mult).

Forward, stepwise jacknife classification scores based on linear discriminants (F-to-enter: 0.15) are shown in Table 1. The model retained two vectors out of four (GM and WM mean concentration) and achieved a $97 \%$ accuracy. 


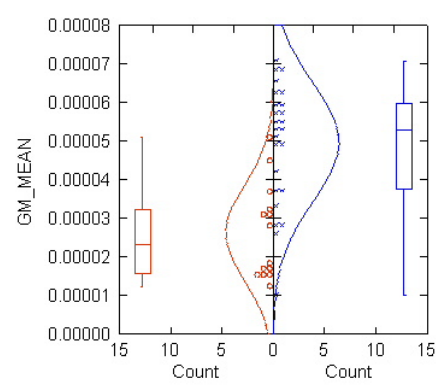

(a)

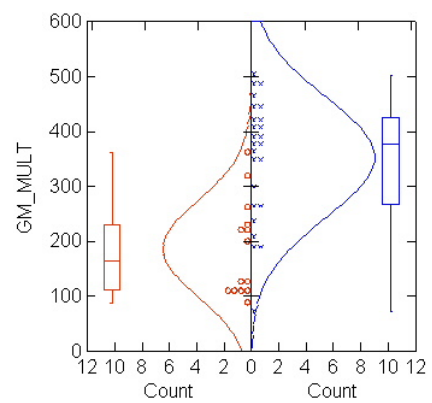

(c)

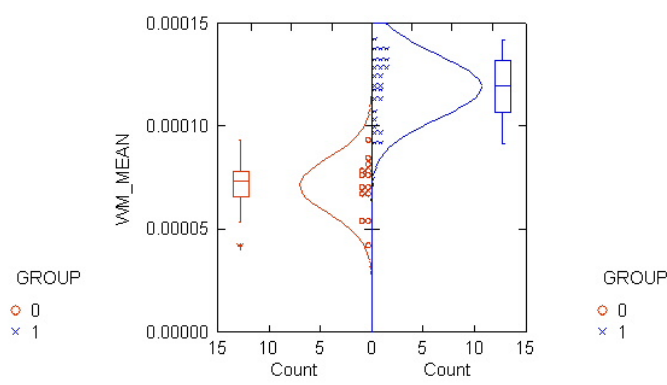

(b)

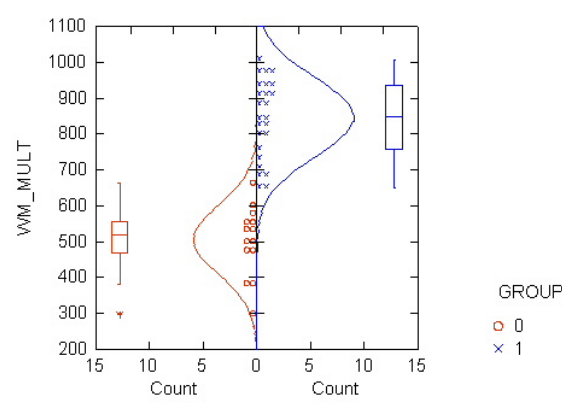

(d)

Fig. 3. (a) and (b) GM and WM mean concentration values, respectively, for all voxels belonging to regions of interest, in individual subjects volumes (c) and (d) GM and WM t-value weighted concentration, respectively, established by multiplying regionsof-interest voxels in individual subjects GM or WM maps with t-value in the corresponding voxel from the between-group comparison. In this figure group 0 represents negative outcome while group 1 represents positive outcome.

Table 1. Forward stepwise jacknife classification (F-to-enter $=0.15$ ) gives best results to classify subjects as belonging to either Positive or Negative surgical outcome groups. True positive results on the Positive-Positive / Negative-Negative diagonal, shown in bold. The analysis reached near-perfect prediction using GM and WM mean concentration.

\begin{tabular}{cccc} 
& Positive & Negative & \% correct \\
\hline Positive & $\mathbf{1 4}$ & 0 & 100 \\
Negative & 1 & $\mathbf{2 4}$ & 96 \\
Total & 15 & 24 & $\mathbf{9 7}$
\end{tabular}

\section{Discussion}

We have been successful in identifying areas of GM and WM concentration differences that were related to surgical outcome in TLE patients having undergone 
selective amygdalo-hippocampectomy using a standard VBM approach. These areas could be refined if we were to use other approaches which improve the accuracy of VBM, such as modulation 10; likewise, an increase in the number of patients would be required as many variables come to play in the post-surgical result. Nevertheless, the fact that there exists statistically significant areas of differences between the two groups is an indication that such information could be used for predictive purposes.

The accuracy of our classifier was excellent. This seems to reflect the straightforward case that patients with poor surgical outcome are more affected by the disease in extra-hippocampal areas, including extra-temporal areas. Their average GM and WM concentration in selected regions of interest are lower than that of positive outcome patients as can be seen from Figure 3

It should be noted that our first analysis is a between-group, voxel-by-voxel comparison of GM or WM concentration whereas the predictive results are individual, region-of-interest based measures. Hence, they do not represent the same information. Clearly, additional subjects, which we are in the process of recruiting, will be required for independent testing and before we can generalize this result in a clinical setting.

\section{Conclusion}

In this work we have set the basis for surgical outcome prediction for temporal lobe epilepsy patients undergoing selective amygdala-hippocampectomy, based on voxel-based morphometry analysis of pre-operative grey and white matter concentration maps. Regions of difference exist between positive and negative outcome patients, and can be used to predict surgical outcome with high accuracy.

Acknowledgments. The authors wish to acknowledge the financial contribution of the Fonds pour la Recherche en Santé du Québec.

\section{References}

1. J. Ashburner et al., Voxel-Based Morphometry: The Methods. NeuroImage, 11:805$821,2000$.

2. N. Bernasconi et al., Voxel-based statistical analysis of grey matter and white matter in patients with unilateral temporal lobe epilepsy. NeuroImage, 19(Suppl. 1): $575,2003$.

3. S.S. Keller et al., Voxel-based morphometric comparison of hippocampal and extrahippocampal abnormalities in patients with left and right hippocampal atrophy. NeuroImage, 16(1):23-31, 2002.

4. K. Woermann et al., Voxel-by-voxel comparison of automatically segmented cerebral gray matter - A rater-independent comparison of structural MRI in patients with epilepsy. NeuroImage, 10(4):373-384, 1999.

5. J. Sled et al., A nonparametric method for automatic correction of intensity nonuniformity in MRI data. IEEE TMI, 17:87-97, 1998. 
6. D. Collins et al., ANIMAL: validation and applications of non-linear registrationbased segmentation IJPRAI, 11:1271-1294, 1997.

7. A. Zijdenbos et al., Morphometric analysis of white matter lesions in MR images: Method and Validation. IEEE TMI, 13: 716-724, 1994.

8. J. Mazziotta et al., A probalistic atlas of the human brain: theory and rationale for its development. NeuroImage, 2:89-101, 1995.

9. K. Worsley et al., Detecting changes in nonisotropic images Human Brain Mapping, 8: 98-101, 1999.

10. C.D. Good et al., A voxel-based morphometric study of ageing in 465 normal adult human brains. NeuroImage, 14:21-36, 2001. 